\title{
Uma Abordagem Automática para Personalização do Pro- cesso de Ensino Baseada em Estilos de Aprendizagem em Sistemas Adaptativos e Inteligentes para Educação a Dis- tância
}

\author{
An Automatic Approach for Customization of Teaching Process Based on Learning \\ Styles in Adaptive and Intelligent Learning Systems
}

\author{
Daydson H. Silva \\ Faculdade de Computação - Universidade Federal de \\ Uberlândia (UFU) \\ Av. João Naves de Ávila, 2121 - Bloco B - Campus Santa \\ Mônica - CEP 38400-902 - Uberlândia-MG \\ daydson.silva@gmail.com
}

\author{
Fabiano A. Dorça \\ Faculdade de Computação - Universidade Federal de \\ Uberlândia (UFU) \\ Av. João Naves de Ávila, 2121 - Bloco B - Campus Santa \\ Mônica - CEP 38400-902 - Uberlândia-MG \\ fabiano@facom.ufu.br
}

\begin{abstract}
Resumo Este artigo apresenta uma nova abordagem para personalização automática do processo de ensino em sistemas adaptativos e inteligentes para educação à distância, levando em conta os estilos de aprendizagem dos estudantes. São mostrados resultados promissores obtidos através de experimentos com um método de adaptação que utiliza uma modelagem de preferências de aprendizagem representadas através de distribuições de probabilidades ao invés de tipos fixos, o que resultou em processos de modelagem do estudante e personalização do processo de ensino não-determinísticos, possibilitando o tratamento das incertezas inerentes ao processo de modelagem de estilos de aprendizagem e adaptação de conteúdo.
\end{abstract}

Palavras-Chave: recomendação de objetos de aprendizagem; modelagem do estudante; estilos de aprendizagem; sistemas tutores inteligentes

\begin{abstract}
This paper presents a new approach for automatic personalization of the teaching process in intelligent and adaptive systems for distance education, taking into account students learning styles. Promising results have been obtained through experiments with a method that uses a learning styles model based on probability distributions instead of using fixed types, which resulted in a non-deterministic student modeling and teaching personalization processes, enabling treatment of the uncertainties inherent to learning styles modeling and content adaptation processes.
\end{abstract}

Keywords: recommendation of learning objects; student modeling; learning styles; intelligent tutoring systems 


\section{Introdução}

A evolução da Educação à Distância (EAD) proporcionou a gradativa substituição dos materiais impressos, distribuídos por correio, aulas exibidas em canais de TV e materiais em áudio ou vídeo disponibilizado por sites desenvolvidos para a Internet com esse fim [1]. Quando esta tecnologia passou a ser utilizada, algumas ferramentas surgiram com o intuito de assessorar aqueles que criam os cursos, tais como Moodle [2], Blackboard [3] e TelEduc [4].

Estas ferramentas, chamadas de Sistemas Gerenciadores de Aprendizagem (ou Learning Management Systems - LMS), apresentam recursos que permitem aos tutores a criação e armazenamento de cursos, bem como o acesso a estes pelos estudantes. Entretanto, a princípio, não disponibilizam possibilidades de personalização do processo de ensino de acordo com as características específicas dos estudantes, o que acredita-se poder causar problemas de aprendizagem [3].

Em face desse problema, o uso de Hipermídia Adaptativa (HA) para promover personalização e facilitar a aprendizagem passou a ser percebido [5], [3]. Há mais de quinze anos, o tema já é discutido, e formas de aplicação de HA neste contexto têm sido analisadas e comparadas [6]. Desta forma, propõe-se a adaptação de conteúdo às características específicas dos estudantes [7], [8]. Algumas pesquisas no campo da HA têm como objetivo encontrar uma forma de modelar as características fundamentais do usuário e utilizar essa informação na apresentação do conteúdo ao estudante [6].

Como resultado de uma pesquisa na área de ensino de engenharia, Felder e Silverman [9] desenvolveram uma proposta para modelagem das características dos Estilos de Aprendizagem (EA) de estudantes por meio de quatro dimensões, com duas possíveis preferências em cada uma.

Estudos atestam que a aprendizagem é facilitada se as estratégias pedagógicas estiverem de acordo com os EA do estudante, e afirmam que utilizar material e atividades instrucionais que vão ao encontro dos EA torna o processo de aprendizagem mais efetivo e a performance do estudante é consideravelmente melhorada [9, 10].

Neste contexto, a personalização automática de conteúdo baseada em EA é um aspecto fundamental em Sistemas Adaptativos e Inteligentes para Educação a Distância (SAIE), sendo esta, atualmente, uma área sob intensa investigação e com grandes perspectivas de aplicação no fornecimento de adaptatividade neste tipo de sis- tema [3].

Seguindo esta linha, este trabalho apresenta uma abordagem automática e dinâmica para personalização do processo de ensino baseada em EA no modelo de Felder e Silverman [9]. Na abordagem proposta neste trabalho, a preferência do estudante é modelada não por tipos fixos, ou categorias determinísticas, mas por distribuições de probabilidades, destinadas a representar a probabilidade de preferência por cada um dos estilos dentro do modelo.

Considerando este aspecto, propõe-se uma abordagem eficiente na detecção automática de EA, que apresenta resultados com alto nível de precisão e baixo custo computacional, principalmente se comparada a outras abordagens analisadas que utilizam regras complexas e técnicas de aprendizagem de máquina.

Ainda, este trabalho apresenta algoritmos de adaptação de conteúdo a partir dos estilos de aprendizagem do estudante. $\mathrm{O}$ processo de adaptação de conteúdo baseado em estilos de aprendizagem é baseado na proposta de [3], mas difere em diversos aspectos. A principal diferença está no fato da abordagem proposta neste trabalho utilizar um modelo do estudante probabilístico, e não determinístico como na abordagem original. Conforme analisa [10], esta característica confere vantagens à abordagem proposta neste trabalho quando comparada a abordagens tradicionais [3],[5],[6],[7],[8],[11] devido ao aspecto de incerteza inerente aos EA e ao próprio processo de modelagem do estudante $[9,10]$.

Os testes realizados apresentaram resultados promissores, demonstrando a viabilidade da utilização de um processo não-determinístico de modelagem do estudante e de recomendação de objetos de aprendizagem baseados na seleção estocástica de combinações de estilos de aprendizagem.

A seção 2 apresenta aspectos teóricos importantes inerentes à abordagem proposta. A seção 3 apresenta a abordagem proposta neste trabalho. A seção 4 apresenta resultados de experimentos realizados com esta abordagem. A seção 5 apresenta as conclusões.

\section{Referencial Teórico}

Nesta sessão, serão apresentados conceitos fundamentais ao entendimento da abordagem proposta neste trabalho. 


\subsection{Estilos de aprendizagem}

Estilos de aprendizagem ditam a maneira pela qual aprendizes percebem, processam, armazenam e recuperam mais eficientemente aquilo que tentam aprender [11]. Além disso, [9], [12], [13], [14] e [15] discorrem sobre a importância do atendimento das preferências de aprendizagem do estudante na facilitação da aprendizagem.

Em [3], apresenta-se vários modelos de estilos de aprendizagem. Uma característica comum dentre eles é o fato de que as pessoas possuem diferentes preferências quanto à forma de absorver e processar conhecimento. São citados, ainda, experimentos que comprovam a redução do tempo necessário para aprender, e elevação da satisfação dos estudantes em sistemas que apresentam adaptatividade baseada em estilos de aprendizagem.

Felder e Silverman [9], na busca pelos problemas que afetavam o desempenho de alunos de engenharia da Universidade do Estado da Carolina do Norte (EUA), analisaram a compatibilidade entre as técnicas de ensino empregadas e as preferências de aprendizagem dos alunos. O resultado do trabalho foi a produção de um detalhado modelo de estilos de aprendizagem, que permite representar a forma como uma pessoa prefere receber e processar novos conhecimentos, de modo a otimizar o processo de aprendizagem. $\mathrm{O}$ modelo apresenta-se composto por quatro dimensões:

- Processamento: O estudante pode preferir lidar com a informação através da experimentação (ativo) ou pela reflexão (reflexivo).

- Percepção: Se o indivíduo tem maior facilidade de percepção do conteúdo pela observação, é considerado sensitivo. Caso perceba melhor através da introspecção, apresenta-se intuitivo.

- Entrada: Caso o aprendizado seja facilitado por informações gráficas, o sujeito é visual, em contraposição à característica verbal daquele que aprende melhor por textos.

- Organização: A pessoa que necessita que o conteúdo esteja previamente disposto em uma ordem específica possui característica sequencial, enquanto aquela que prefere percorrer livremente $o$ conteúdo a ser estudado é caracterizada como global.

A aplicação deste modelo tornou-se mais acessível após a criação do Index of Learning Styles Questionnaire (ILSQ) [12]. Aplica-se quarenta e quatro questões objeti- vas (sendo que cada conjunto de onze questões é dedicado a uma das quatro dimensões do modelo). Para cada questão existem duas respostas possíveis. Cada resposta reforça a preferência para um extremo da dimensão à qual a questão é dedicada. Atribui-se o valor de -1 para uma resposta com tendência a um extremo e o valor de +1 caso contrário. $\mathrm{O}$ resultado é um número ímpar no intervalo $[-11,+11]$.

Considerando o módulo do valor obtido, a tendência dentro da dimensão avaliada pode ser balanceada, para os valores 1 ou 3, moderada, se o valor for 5 ou 7 , ou forte, para resultados 9 ou 11 [13]. Esquematicamente, cada dimensão do modelo pode ser visualizada como mostrado na Figura 1.

\begin{tabular}{|c|c|c|c|c|c|c|c|c|c|c|c|}
\hline-11 & -9 & -7 & -5 & -3 & -1 & 1 & 3 & 5 & 7 & 9 & 11 \\
\hline \multicolumn{3}{|c|}{ FORTE } & MODERADO & & BaLANCEAdO & & MODERADO & \multicolumn{2}{c|}{ FORTE } \\
\hline \multicolumn{3}{|c|}{ PÓlO A } & & & \multicolumn{3}{|c|}{ PÓlO B } \\
\hline
\end{tabular}

Figura 1: Representação esquemática de uma dimensão do modelo de Felder e Silverman.

\subsection{Hipermídia adaptativa}

Segundo Wu [16], os sistemas de hipermídia adaptativa funcionam, basicamente, armazenando um modelo de usuário (MU) o qual contém as características consideradas relevantes do usuário e, a partir destas, gerando adaptação de conteúdo ou adaptação de navegação de hipermídia.

No caso de adaptação de conteúdo com intuito educacional, em [3] apresenta-se o conceito de cursos disponibilizados através de páginas web, montadas com estruturas modulares chamadas de Learning Objects (LO), ou Objetos de Aprendizagem (OA). Estes são estruturas destinadas a armazenar partes do conteúdo de uma lição, como o comentário que introduz a lição, o conteúdo propriamente dito, que apresenta a teoria a ser aprendida, a conclusão, as avaliações, exercícios, exemplos, animações e outros. A partir daí, os autores vislumbram a possibilidade de se produzir adaptatividade através da montagem de páginas, utilizando os módulos em uma sequência que prioriza os OA's que estejam mais adequados ao estudante a ser atendido.

Neste sentido, Brusilovski [17] cita a existência de trabalhos envolvendo OA's que contenham metadados (informações sobre o conteúdo dos OA's), os quais são imprescindíveis para a recuperação automática deste conteúdo, e assevera a importância do desenvolvimento de 
"material educacional mais inteligente que possa ser naturalmente utilizado e reutilizado pelos sistemas de hipermídia educacional adaptativa" [17].

Então, outros ramos de pesquisas surgiram no intuito de se obter a melhor forma de mapear o conteúdo dos domínios a serem lecionados. Neste sentido, Munoz e Oliveira [18] citam o Learning Objects Metadata Standard, padrão proposto pelo IEEE (Institute of Electrical and Electronics Engineers) largamente utilizado na criação de metadados para OAs.

\subsection{Recomendação automática de conteúdo}

Para a implementação da personalização de conteúdo, vários métodos vêm sendo propostos, como é o caso do método apresentado por Paredes e Rodrigues [13]. A proposta consiste apenas na ordenação entre conceitos de uma lição e seus respectivos exemplos, levando em consideração a dimensão Percepção do estudante. Outros métodos mais sofisticados podem ser encontrados, os quais contam com várias regras de adaptatividade baseadas em associações propostas entre estilos de estudantes e tipos de objetos de aprendizagem.

Em [3] apresenta-se um método para recomendação de conteúdo a partir dos estilos de aprendizagem do estudante, chamado "Mecanismo Flexível". O método apresenta técnicas de adaptação de conteúdo com base nas características do estudante presentes no modelo proposto por Felder e Silverman [9].

Tal método propõe que os conceitos a serem estudados sejam apresentados através de determinados tipos de OA's, considerando-se os estilos de aprendizagem específicos de um estudante. Os tipos de OA's considerados pelo Mecanismo Flexível são:

- Comentários: apresentam ao aprendiz uma visão geral do conteúdo da lição.

- Objetos de Conteúdo: são usados para apresentar o conteúdo propriamente dito do curso.

- Questionários de reflexão (quiz): incluem uma ou mais questões cujas respostas ainda estão em aberto, que têm o intuito de incentivar a reflexão.

- Testes de auto-avaliação: incluem questões para que o próprio aluno avalie seu conhecimento, com feedback imediato.

- Fórum de discussão: possibilita ao estudante discutir o conteúdo com colegas ou instrutores.
- Leitura adicional: provê fontes extras de leitura a respeito do conteúdo da lição.

- Animação: demonstra o conceito do curso em formato de multimídia animada.

- Exercício: provê espaço para praticar o conteúdo aprendido.

- Exemplo: ilustra os conceitos da teoria de forma concreta.

- Aplicações na vida real: demonstram como o material pode relacionar-se com as situações do dia-a-dia.

- Conclusão: resume e encerra o conteúdo.

- Avaliação: reúne as tarefas necessárias à avaliação do aprendizado da lição.

Um dos motivos de ser chamado de flexível devese ao fato de exigir apenas que exista um OA do tipo Objeto de Conteúdo, independente de sua forma de apresentação. Entretanto, quanto mais objetos estiverem disponíveis, melhor será o efeito de adaptatividade proporcionada pelo método. Considerando que, dificilmente, uma lição possa ser ministrada sem um núcleo, tal exigência não se torna empecilho para a grande maioria de lições que se queira disponibilizar em um curso.

A aplicação do método, que se dá em dois estágios, presume a existência de uma lição e um modelo de estudante. A lição deve ser composta por, no mínimo um OA do tipo Objeto de Conteúdo que possa exibir o seu conteúdo teórico. Para cada lição, todos os OAs disponíveis serão utilizados. O primeiro estágio é a anotação, onde os objetos, cujos tipos têm afinidade com o estilo do aluno são marcados como recomendados, da mesma forma que os objetos considerados essenciais: comentário inicial, conteúdo, conclusão e avaliação.

Para a compreensão do segundo estágio do método, é necessário ter em mente a estrutura da página a ser montada, que está representada na Figura 2. Na referida estrutura, o objeto "Conteúdo" sempre existirá por ser a única exigência para que o método seja aplicado. Os objetos "Comentário" e "Avaliação", caso existam, serão o primeiro e o último OA da lição, respectivamente. 


\begin{tabular}{|c|}
\hline Comentário \\
\hline [Área 1] \\
\hline Conteúdo \\
\hline [Área 2] \\
\hline Avaliação \\
\hline
\end{tabular}

Figura 2: Estrutura da página que representa uma lição adaptada pelo Mecanismo Flexível.

O segundo estágio é a ordenação, que determinará a posição dos demais OA's dentro da página. Em [3] citase uma série de associações entre os estilos de aprendizagem e os tipos de OA's considerados pelo método. Tais associações, bem como a recomendação de aparecerem na Área 1 (A1) ou na Área 2 (A2) estão representadas na Tabela 1. Para ambas as áreas, os OA's são incluídos na ordem decrescente de intensidade de afinidade com o modelo de estudante utilizado.

De acordo com [3], na área 1 é recomendada a uti-

\begin{tabular}{|c|c|c|c|c|c|c|c|c|}
\hline \multirow{3}{*}{ Tipo de Objeto } & \multicolumn{8}{|c|}{ PERFIL DO ESTUDANTE } \\
\hline & \multicolumn{2}{|c|}{ Processamento } & \multicolumn{2}{|c|}{ Percepção } & \multicolumn{2}{|c|}{ Entrada } & \multicolumn{2}{|c|}{ Organização } \\
\hline & Ativo & Reflexivo & Sensitivo & Intuitivo & Visual & Verbal & Sequencial & Global \\
\hline Questionário de reflexão & - & $\mathrm{A} 2$ & - & $\mathrm{A} 2$ & - & - & - & - \\
\hline Teste de auto-avaliação & A1 & - & $\mathrm{A} 2$ & - & - & - & - & - \\
\hline Fórum de discussão & $\mathrm{A} 2$ & - & - & - & - & $\mathrm{A} 2$ & - & - \\
\hline Leitura adicional & - & $\mathrm{A} 2$ & - & $\mathrm{A} 2$ & - & $\mathrm{A} 2$ & - & - \\
\hline Animação & $\mathrm{A} 1$ & - & $\mathrm{A} 1$ & - & $\mathrm{A} 1$ & - & - & - \\
\hline Exercício & A1 & - & $\mathrm{A} 2$ & $\mathrm{~A} 1$ & - & - & - & - \\
\hline Exemplo & - & $\mathrm{A} 2$ & $\mathrm{~A} 1$ & - & - & - & - & $\mathrm{A} 2$ \\
\hline Aplicações na vida real & - & - & A1 & - & - & - & - & A2 \\
\hline
\end{tabular}

Tabela 1. Adequação e posicionamento dos OAs às áreas 1 ou 2, conforme perfil do estudante.

\begin{tabular}{|l|c|c|c|c|c|c|c|c|}
\hline \multirow{3}{*}{ Tipo de Objeto } & \multicolumn{9}{|c|}{ PERFIL DO ESTUDANTE } \\
\cline { 2 - 10 } & \multicolumn{2}{|c|}{ Processamento } & \multicolumn{2}{|c|}{ Percepcão } & \multicolumn{2}{c|}{ Entrada } & \multicolumn{3}{|c|}{ Organização } \\
\cline { 2 - 9 } & Ativo & Reflexivo & Sensitivo & Intuitivo & Visual & Verbal & Sequencial & Global \\
\hline Conclusão & Fim & Início & Fim & Fim & - & Fim & Fim & Início \\
\hline
\end{tabular}

Tabela 2. Posicionamento do objeto Conclusão na área 2, conforme perfil do estudante.

$\mathrm{Na}$ abordagem proposta neste trabalho, a preferência por cada estilo de aprendizagem é armazenada no modelo do estudante como um valor real lização de, no máximo, dois tipos de OAs, para não sobrecarregar o aprendiz com atividades antes do contato com o conteúdo central da lição. Objetos do tipo conclusão figuram sempre no início ou no fim da área 2 , a depender do perfil do estudante, como visto na Tabela 2, enquanto as avaliações, invariavelmente, encerram a estrutura da página.

\section{Abordagem proposta}

Esta seção apresenta uma abordagem automática e dinâmica para personalização do processo de ensino. Esta abordagem é baseada no modelo de Felder e Silverman para EA e na inter-relação entre este modelo e os tipos de conteúdos apresentados pelo mecanismo flexível, de acordo com as Tabelas 1 e 2 .

A abordagem proposta tem ainda como pilar um modelo do estudante baseado em distribuições de probabilidades, o que permite tratar as incertezas inerentes ao processo de ensino. 
com isto, passamos a representar as preferências do estudante como distribuições de probabilidades que são armazenadas diretamente no modelo do estudante.

Desta forma, tem-se um modelo do estudante probabilístico em que estilos de aprendizagem são tratados pelo sistema como probabilidades, e não como certezas, e o FSLSM permite isto. A Tabela 3 apresenta um exemplo deste modelo. Este modelo ue inova em, pelo menos, três aspectos:

- Fornece um valor probabilístico como representação da tendência do estudante para cada estilo de aprendizagem dentro de cada dimensão do modelo, tornando mais refinado e detalhado o modelo do estudante, e então tornando sua representação mais realista.

- A modelagem é automática e dinâmica, obtida iterativamente através da análise do resultado da avaliação de desempenho do estudante e dos tipos de objetos que lhe foram apresentados, eliminando-se a necessidade de aplicação de questionários, o que traz diversos benefícios [10].

- Não afirma deterministicamente que o estilo que possui uma probabilidade maior seja efetivamente o preferido pelo estudante. Sugere apenas uma possibilidade de preferência, mas admite que o estilo de menor probabilidade possa ser o correto. Desta forma, permite através de um processo iterativo que inconsistências no modelo do estudante sejam corrigidas.

Desta forma, considerando o aspecto probabilístico do modelo, o exemplo fornecido pela Tabela 3 representa um estudante com $35 \%$ de probabilidade de preferência pelo estilo ativo e $65 \%$ de probabilidade de preferência pelo estilo reflexivo; $17 \%$ de probabilidade de preferência pelo estilo sensitivo e $83 \%$ de probabilidade de preferência pelo estilo intuitivo; $89 \%$ de probabilidade de preferência pelo estilo visual e $11 \%$ de probabilidade de preferência pelo estilo verbal; $84 \%$ de probabilidade de preferência pelo estilo sequencial e $16 \%$ de probabilidade de preferência pelo estilo global. Então, pode-se considerar que este estudante provavelmente é Reflexivo, Intuitivo, Visual e Sequencial.

\begin{tabular}{|c|c|c|c|c|c|c|c|}
\hline \multicolumn{7}{|c|}{ Estilos de Aprendizagem Probabilisticos (EAp) } \\
\hline Processamento & \multicolumn{2}{|c|}{ Percepção } & \multicolumn{2}{c|}{ Entrada } & \multicolumn{2}{c|}{ Organização } \\
\hline Ativo & Reflex. & Sens. & Intuit. & Visual & Verbal & Seq. & Global \\
\hline 0,35 & 0,65 & 0,17 & 0,83 & 0,89 & 0,11 & 0,84 & 0,16 \\
\hline
\end{tabular}

Tabela 3. Modelo do estudante probabilístico em que estilos de aprendizagem são tratados pelo sistema como probabilidades

A Figura 3 apresenta dois diferentes estudantes modelados utilizando esta abordagem. Neste caso, o estudante (a) é modelado com as seguintes probabilidades de preferências: $100 \%$ para Reflexivo, $65 \%$ para Intuitivo, $68 \%$ para Visual e $82 \%$ para Global. O estudante (b) é modelado com as seguintes probabilidades de preferências: $85 \%$ para Reflexivo, $65 \%$ para Sensitivo, $82 \%$ para Visual e $68 \%$ para Global.

Desta forma, é importante notar que ambos os estudantes possuem uma tendência maior pelas mesmas preferências, porém com probabilidades diferentes, levando-se em consideração que estas tendências são probabilísticas, e não determinísticas, podendo resultar em processos adaptativos diferentes.

Caso seja utilizado um instrumento para a identificação de estilos de aprendizagem compatível com o FSLSM, como o Index of Learning Styles Questionnaire [10], o modelo pode ser inicializado através dos dados obtidos pelo questionário, fazendo-se a conversão considerando-se a proporcionalidade das respostas pontuadas para cada estilo de aprendizagem.

Esta conversão é dada por (1), que divide a quantidade de respostas favoráveis a um estilo $(A i)$ pelo total de respostas dentro da dimensão, que é $11 \mathrm{em}$ todas as dimensões (totalizando 44 questões).

$$
P \square \equiv \frac{A i}{11}
$$




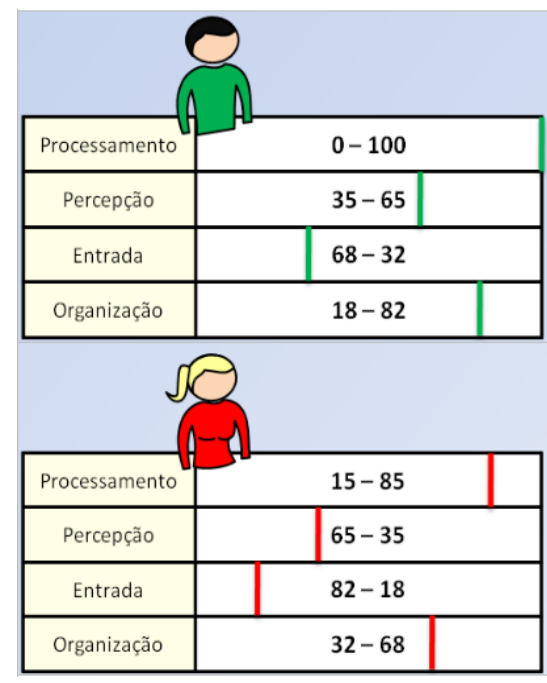

Figura 3. Perfis similares, mas com probabilidades diferentes

Por exemplo, caso um estudante tenha respondido 3 questões favoráveis ao estilo Ativo e 8 questões favoráveis ao estilo Reflexivo, na dimensão Processamento, tem-se:

$$
\begin{aligned}
& P \sqsubset A \sqsubseteq \frac{3}{11}=0.28 \\
& P \square R \equiv \frac{8}{11}=0.72
\end{aligned}
$$

em que $P(A)$ representa a probabilidade e preferência pelo estilo ativo e $P(R)$ representa a probabilidade de preferência pelo estilo reflexivo.

Este modelo traz algumas vantagens importantes, como por exemplo, levar em consideração que é impossível saber o quão consistente é o resultado obtido em um questionário de auto-avaliação de estilos de aprendizagem, e um sistema que use um modelo determinístico fica incapacitado em detectar inconsistências no modelo do estudante, já que não leva em consideração outras combinações de estilos de aprendizagem que não aquela previamente obtida pelo questionário.

Com base neste modelo de probabilidades, a cada seção de aprendizagem do curso, o sistema seleciona estocasticamente uma combinação de estilos de aprendizagem cujos elementos são combinados de acordo com as probabilidades de preferência do estudante.
Considerando as 4 dimensões do FSLSM tem-se 16 possíveis combinações de estilos de aprendizagem.

Neste contexto, uma grande vantagem desta abordagem é considerar estocasticamente, durante o processo de aprendizagem, todas as combinações de estilos de aprendizagem de acordo com as probabilidades de preferências do estudante armazenadas no modelo do estudante, que podem estar inconsistentes ou variar ao longo do tempo em decorrência de algum aspecto, conforme discutido anteriormente.

Considerando os EAp do estudante, a probabilidade $\mathrm{P}$ de pertinência do estudante a uma categoria pedagógica é dada por (2), que é a mesma de uma CEA ser selecionada durante uma seção de aprendizagem, que é igual à probabilidade de preferência do estudante pela CEA. Em (2), a, b, c, e d representam os EA que compõem a CEA considerada.

$$
\mathrm{P}(\mathrm{a}, \mathrm{b}, \mathrm{c}, \mathrm{d})=\mathrm{P}(\mathrm{a}) \times \mathrm{P}(\mathrm{b}) \times \mathrm{P}(\mathrm{c}) \times \mathrm{P}(\mathrm{d})
$$

Desta forma, de acordo com (2), a probabilidade de preferência do estudante pela CEA (A,S,Vi,Seq), considerando-se os EAp apresentados na Tabela 3, é dada por (tal que A = Ativo; $\mathrm{S}=$ Sensitivo; Vi = Visual; $\mathrm{Seq}=$ Sequencial):

$$
\begin{aligned}
& \mathrm{P}(\mathrm{A} ; \mathrm{S} ; \mathrm{Vi} ; \mathrm{Seq})=\mathrm{P}(\mathrm{A}) \times \mathrm{P}(\mathrm{S}) \times \mathrm{P}(\mathrm{Vi}) \times \mathrm{P}(\mathrm{Seq}) \\
& \mathrm{P}(\mathrm{A} ; \mathrm{S} ; \mathrm{Vi} ; \mathrm{Seq})=0,35 \times 0,17 \times 0,89 \times 0,84=0,045
\end{aligned}
$$

Desta forma, considerando o modelo do estudante apresentado na Tabela 3, tem-se a distribuição de probabilidades apresentada na Tabela 4.

Para decidir como os estilos de aprendizagem probabilísticos do estudante são atualizados, leva-se em consideração qual combinação de estilos de aprendizagem foi aplicada durante uma seção de aprendizagem.

Ao longo do processo de modelagem automática de estilos de aprendizagem, o modelo do estudante é atualizado da seguinte maneira: ao se detectar dificuldades de aprendizagem ou desempenho insatisfatório em uma seção de aprendizagem, os estilos de aprendizagem presentes na combinação de estilos de 
aprendizagem selecionada são decrementados no modelo do estudante, considerando-se uma possível inconsistência.

Em contrapartida, aqueles estilos de aprendizagem ausentes na combinação de estilos de aprendizagem são incrementados no modelo do estudante, reforçando-se sua preferência pelo estudante e, portanto, suas chances de estar presente em uma combinação de estilos de aprendizagem em uma próxima seção de aprendizagem, considerando-se que, possivelmente, o não atendimento a estas preferências geraram dificuldades de aprendizagem.

\begin{tabular}{|l|l|}
\hline CEA & Distribuição de Probabilidades \\
\hline $\mathrm{P}(\mathrm{A}, \mathrm{S}, \mathrm{Vi}, \mathrm{Seq})$ & $0.35 \times 0.17 \times 0.89 \times 0.84=0.045$ \\
\hline $\mathrm{P}(\mathrm{A}, \mathrm{S}, \mathrm{Vi}, \mathrm{G})$ & $0.35 \times 0.17 \times 0.89 \times 0.16=0.008$ \\
\hline $\mathrm{P}(\mathrm{R}, \mathrm{S}, \mathrm{Vi}, \mathrm{Seq})$ & $0.65 \times 0.17 \times 0.89 \times 0.16=0.016$ \\
\hline $\mathrm{P}(\mathrm{R}, \mathrm{S}, \mathrm{Vi}, \mathrm{G})$ & $0.35 \times 0.17 \times 0.11 \times 0.84=0.005$ \\
\hline $\mathrm{P}(\mathrm{A}, \mathrm{S}, \mathrm{Ve}, \mathrm{Seq})$ & $0.35 \times 0.17 \times 0.11 \times 0.16=0.002$ \\
\hline $\mathrm{P}(\mathrm{A}, \mathrm{S}, \mathrm{Ve}, \mathrm{G})$ & $0.65 \times 0.17 \times 0.11 \times 0.84=0.010$ \\
\hline $\mathrm{P}(\mathrm{R}, \mathrm{S}, \mathrm{Ve}, \mathrm{Seq})$ & $0.65 \times 0.17 \times 0.11 \times 0.16=0.003$ \\
\hline $\mathrm{P}(\mathrm{R}, \mathrm{S}, \mathrm{Ve}, \mathrm{G})$ & $0.35 \times 0.83 \times 0.89 \times 0.84=0.217$ \\
\hline $\mathrm{P}(\mathrm{A}, \mathrm{I}, \mathrm{Vi}, \mathrm{Seq})$ & $0.35 \times 0.83 \times 0.89 \times 0.16=0.043$ \\
\hline $\mathrm{P}(\mathrm{A}, \mathrm{I}, \mathrm{Vi}, \mathrm{G})$ & $0.65 \times 0.83 \times 0.89 \times 0.84=0.403$ \\
\hline $\mathrm{P}(\mathrm{R}, \mathrm{I}, \mathrm{Vi}, \mathrm{Seq})$ & $0.65 \times 0.83 \times 0.89 \times 0.16=0.076$ \\
\hline $\mathrm{P}(\mathrm{R}, \mathrm{I}, \mathrm{Vi}, \mathrm{G})$ & $0.35 \times 0.83 \times 0.11 \times 0.84=0.026$ \\
\hline $\mathrm{P}(\mathrm{A}, \mathrm{I}, \mathrm{Ve}, \mathrm{Seq})$ & $0.35 \times 0.83 \times 0.11 \times 0.16=0.005$ \\
\hline $\mathrm{P}(\mathrm{A}, \mathrm{I}, \mathrm{Ve}, \mathrm{G})$ & $0.65 \times 0.83 \times 0.11 \times 0.84=0.049$ \\
\hline $\mathrm{P}(\mathrm{R}, \mathrm{I}, \mathrm{Ve}, \mathrm{Seq})$ & \\
\hline $\mathrm{P}(\mathrm{R}, \mathrm{I}, \mathrm{Ve}, \mathrm{G})$ & $\mathrm{S})$ \\
\hline Soma das probabilidades & \\
\hline
\end{tabular}

Tabela 4. Distribuição de Probabilidades das possíveis Combinações de Estilos de Aprendizagem

O desempenho do estudante é considerado insatisfatório quando não atinge um limiar $(m)$, que pode ser particular a cada LMS, ou até mesmo a um curso específico dentro de um sistema. Para decidir como o modelo do estudante deve ser alterado, foi implementado um conjunto de regras de produção, do tipo se...então, que levam em consideração os estilos de aprendizagem probabilísticos e a combinação de estilos de aprendizagem considerada durante a seção de aprendizagem.

O reforço é sempre aplicado a todos os estilos de aprendizagem probabilísticos no modelo do estudante, já que o sistema não sabe onde estão as inconsistências no modelo do estudante que levaram ao problema de aprendizagem. Considerando-se que cada dimensão do FSLSM possui dois estilos de aprendizagem, representados por $A$ e $B$ nas regras a seguir, tem-se:

$$
\begin{aligned}
&(\mathrm{PFM}<\mathrm{m}) \wedge(\mathrm{CEA}[\mathrm{i}]=\mathrm{A}) \rightarrow \\
& \mathrm{EAp}[\mathrm{i}] \mathrm{A}:=\mathrm{EAp}[\mathrm{i}] \mathrm{A}-\alpha \times \mathrm{R}, \\
& \mathrm{EAp}[\mathrm{i}] \mathrm{B}:=\mathrm{EAp}[\mathrm{i}] \mathrm{B}+\alpha \times \mathrm{R} . \\
&(\mathrm{PFM}<\mathrm{m}) \wedge(\mathrm{CEA}[\mathrm{i}]=\mathrm{B}) \rightarrow \\
& \mathrm{EAp}[\mathrm{i}] \mathrm{A}:=\mathrm{EAp}[\mathrm{i}] \mathrm{A}+\alpha \times \mathrm{R}, \\
& \mathrm{EAp}[\mathrm{i}] \mathrm{B}::=\operatorname{EAp}[\mathrm{i}] \mathrm{B}-\alpha \times \mathrm{R} .
\end{aligned}
$$

em que:

$1 \quad \operatorname{EAp}[i]_{\mathrm{A}}$ representa o valor do EAp do estudante na dimensão $i$ para o estilo de aprendizagem $A$, para $i=$ $1 \ldots 4$.

2 EAp $[i]_{\text {в }}$ representa o valor do Eap do estudante na dimensão $i$ para o estilo de aprendizagem $B$, para $i=$ $1 \ldots 4$.

$3 \mathrm{CEA}[i]$ representa o elemento que constitui a combinação de estilos de aprendizagem considerando a dimensão $i, \operatorname{com} i=1 \ldots 4$.

4 R é o reforço a ser aplicado nos na modificação dos estilos de aprendizagem probabilísticos do estudante.

$5 \alpha$ é a taxa de aprendizagem.

Informações sobre como é realizado o cálculo do reforço, sobre a taxa de aprendizagem e detalhes sobre a eficiência da política implementada por estas regras comparada a outras podem ser obtidas em [15].

O Algoritmo 1 implementa o processo de aprendizagem considerando este modelo para modelagem automática dos estilos de aprendizagem dos estudantes, possibilitando ao sistema a seleção de estratégias de aprendi- 
zagem mais apropriadas.

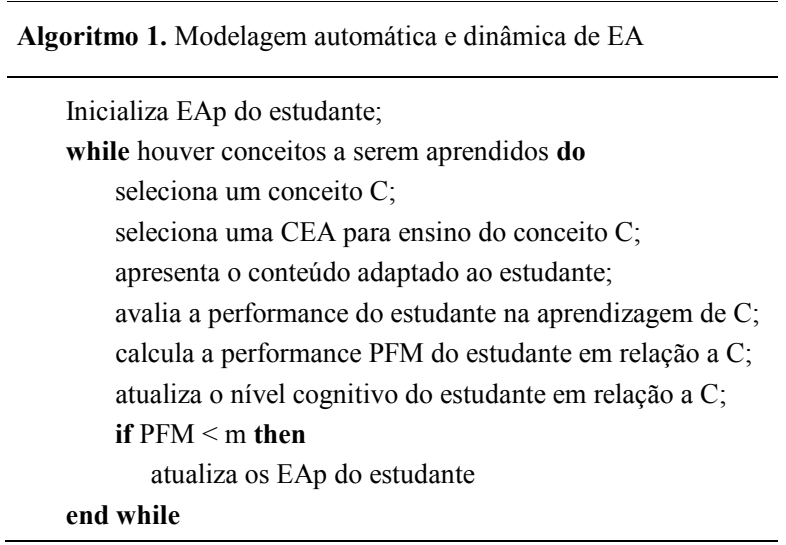

Para a obtenção da sequência de conteúdos que melhor se adapta às preferências do estudante, é atribuído um peso a cada objeto disponível para determinada lição, o qual resulta da soma dos valores dos EAp cujos EAs pertençam à CEA selecionada durante determinada seção de aprendizagem.

Como exemplo, se um objeto do tipo Leitura Adicional está disponível em uma lição e a CEA gerada possui os EA Reflexivo com EAp = 0,8 e Intuitivo com EAp $=0,7$, então, o peso do objeto na lição é de 1,5 .

O resultado final do processo de adaptatividade é, inicialmente, uma página em formato HTML que contém links para os diversos conteúdos recomendados, apresentados na ordem decrescente de pesos. O Algoritmo 2 implementa este processo, levando em consideração as informações apresentadas na Tabela 1.

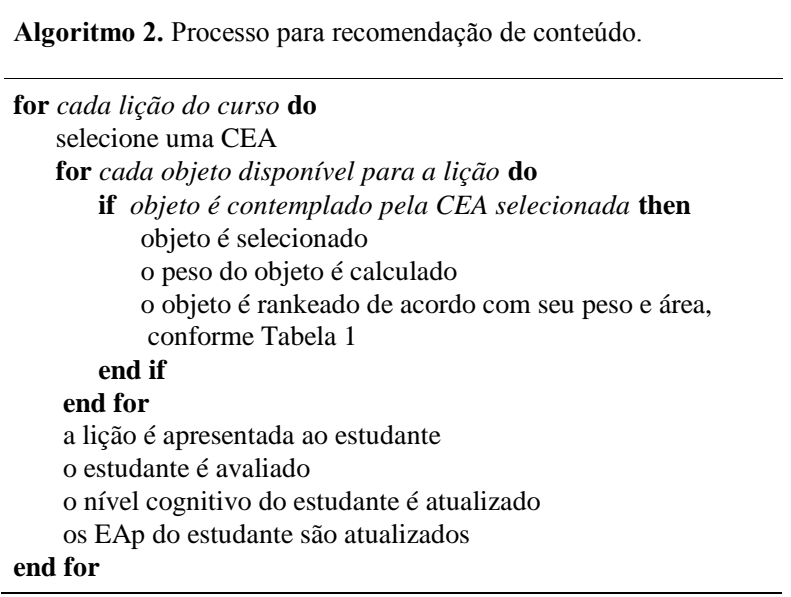

É possível notar que se um conteúdo é recomendado para diferentes áreas, dependendo do EA, então, ele será posicionado na área correspondente ao maior EAp. Por exemplo, se um conteúdo do tipo Teste de autoavaliação é contemplado pela CEA selecionada e recomendado para a área 1 (EA Ativo) com peso EAp $=0,35$, e para a área 2 (EA Sensitivo) com peso EAp $=0,17$, então, este objeto será posicionado na área 1 , com peso 0,52. Além disto, para posicionamento de conteúdos do tipo Conclusão, baseando-se na Tabela 2, utiliza-se o Algoritmo 3.

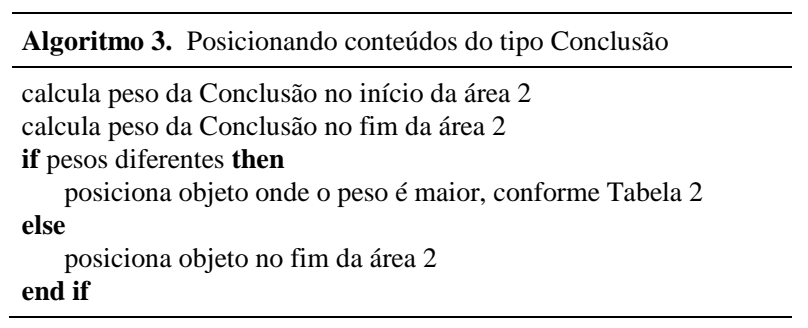

No Algoritmo 3, é importante notar que caso os pesos para posicionamento da conclusão no início e no fim da área 2 sejam iguais, este conteúdo é posicionado no fim da área 2 , por esta ser a recomendação dada para a maioria dos estilos, de acordo com a Tabela 2.

Com intuito de se testar esta abordagem, isolando toda a complexidade relacionada a avaliação de performance do estudante, desenvolveu-se um modelo cognitivo para simulação da performance do estudante em um processo de ensino-aprendizagem simulado.

O objetivo principal foi observar como os estilos de aprendizagem do estudante são modificados em decorrência do resultado de sua performance à medida que o curso evolui, e então testar, avaliar e validar a política para detecção de estilos de aprendizagem apresentada.

Este modelo considera importantes aspectos relacionados ao impacto de estilos de aprendizagem no processo de aprendizagem, levantados por [9], baseado em extensa experimentação em uma população de estudantes de cursos superiores de engenharia.

O principal aspecto deste modelo é que quando os estilos de aprendizagem reais de um estudante são 
atendidos pela combinação estilos de aprendizagem selecionada, o processo de aprendizagem tende a se tornar mais fácil, e a probabilidade de sucesso é maior.

Desta forma, este modelo considera um aumento na dificuldade quando os estilos de aprendizagem reais do estudante não são atendidos pelo sistema. Então, o modelo infere o grau de dificuldade a ser enfrentado pelo estudante durante uma seção de aprendizagem elevando a probabilidade de falha.

Considera-se ainda que o processo de aprendizagem é não-determinístico, e pode ser influenciado por muitos fatores além dos estilos de aprendizagem, de forma que um processo de ensino inadequado pode contribuir para a ocorrência de falha, mas não pode determina-la [9].

Outro aspecto importante do modelo é que preferências fortes produzem efeitos negativos mais notórios no processo de aprendizagem quando não são satisfeitas, conforme atesta [9]. Neste contexto, o modelo calcula a quantidade de divergências $(d)$ entre os estilos de aprendizagem reais do estudante e a combinação de estilo de aprendizagem selecionada pelo sistema, e a utiliza no cálculo de um fator de dificuldade $(D)$ dado por (2).

$$
D=d+\beta+\gamma
$$

tal que:

- $d$ é a quantidade de divergências entre a combinação de estilos de aprendizagem selecionada e os estilos de aprendizagem reais do estudante, estando no intervalo $[0,4]$.

- $\quad \beta$ é avaliado como um número aleatório entre $[0,1]$ sempre que uma preferência forte do estudante não for satisfeita pelo sistema. Caso contrário, seu valor é 0 .

- $\quad \gamma$ é avaliado como um número aleatório entre [0, $1-\beta]$ sempre que uma preferência moderada do estudante não for satisfeita pelo sistema. Caso contrário, seu valor é 0 .

Preferências leves ou balanceadas não elevam o fator de dificuldade. Desta forma, $\beta$ e $\gamma$ permitem ao modelo considerar a força das preferências e sua influência no processo de aprendizagem.
O fator de dificuldade $D$ é utilizado no cálculo da performance $(P F M)$ do estudante, conforme apresenta (3), estando seu valor no intervalo $[0,100]$.

$$
\mathrm{PFM}=\mathrm{M}-(\mathrm{D} \times \mathrm{K} \times \alpha)
$$

tal que:

- $\quad M$ é o valor máximo da performance, neste caso $M=100$.

- $\quad D$ é o fator de dificuldade dado por (2), cujo valor está no intervalo $[0,5]$.

- $K$ é uma constante, e possibilita que o valor de PFM seja 0 se $D$ e $\alpha$ assumirem seus valores máximos. Desta forma, $K=$ 20.

- $\quad \alpha$ é um multiplicador, dado por um número aleatório no intervalo $[0,1]$, e representa um fator de dificuldade não determinístico, considerando-se que não apenas estilos de aprendizagem, mas outros fatores exercem influência na performance do estudante, conforme mencionado anteriormente.

Desta forma, este modelo permite inferir a performance de estudantes em um ambiente simulado, e consequentemente a evolução do nível cognitivo, de forma probabilística, em um processo de ensinoaprendizagem, criando o ambiente propício para experimentação de novos sistemas de e-learning, no que tange à modelagem de estilos de aprendizagem do estudante e ao fornecimento de experiências de ensino personalizadas, considerando adaptatividade baseada em estilos de aprendizagem.

É particularmente interessante conseguir conhecer o comportamento e funcionamento destes novos sistemas antes de testá-los com estudantes reais, já que testes com estudantes reais são mais complexos, custosos e demorados. Desta forma, a aplicação de simulação computacional em novos modelos podem agilizar bastante o processo de desenvolvimento e testes.

A próxima seção apresenta resultados de experimentos realizados com a abordagem proposta nesta seção, com o intuito de testa-la e valida-la. 


\section{Experimentos e Resultados}

Esta seção apresenta resultados de experimentos, em que considerou-se o valor da performance mínima esperada $m=60,0 \%$ (padrão adotado na maioria dos processos de ensino). Foi considerado um conjunto de 30 conceitos a serem aprendidos pelo estudante simulado, onde em cada conceito o estudante deve percorrer 6 níveis. Desta forma, o nível cognitivo do estudante evolui por seis níveis, dispostos em uma hierarquia, do menos complexo para o mais complexo.

Portanto, o processo de aprendizagem simulado nesses experimentos deve ter, pelo menos, 180 sessões de aprendizagem (ou iterações) a fim de alcançar todas as metas de aprendizagem (30 conceitos $\times 6$ níveis cognitivos em cada conceito $=180$ ). A execução de um experimento é finalizada quando o estudante atinge todos os objetivos de aprendizagem especificados. A fim de validar a abordagem proposta, foram observadas duas variáveis nos experimentos:

- consistência: os estilos de aprendizagem probabilísticos efetivamente convergem para os estilos de aprendizagem reais do estudante durante o processo de aprendizagem?

- eficiência: os estilos de aprendizagem probabilísticos convergem em direção aos estilos de aprendizagem reais do estudante em tempo razoável, isto é, estilos de aprendizagem probabilísticos tornam-se consistentes no início do processo de aprendizagem?

Neste momento, é importante notar que sem a utilização de simulação seria difícil a validação da abordagem proposta. Isto porque ao se testar a abordagem proposta em processos de aprendizagem reais, com estudantes reais, seria impossível conhecer com exatidão seus estilos de aprendizagem reais, em decorrência dos motivos apontados anteriormente sobre a utilização de auto-concepções do estudante e sobre a utilização de processos e técnicas determinísticas na inferência de seus estilos de aprendizagem.

Desta forma, seria impossível medir o nível de consistência do modelo do estudante obtido durante o processo de aprendizagem, impossibilitando-nos medir duas variáveis muito importantes na validação da abordagem proposta: a eficiência em relação ao tempo despendido na detecção de estilos de aprendizagem, assim como na correção de inconsistências existentes no modelo do estudante.

Este é o motivo principal pelo qual este trabalho foca na análise da viabilidade da abordagem proposta sob uma ótica simulada antes de nos propor a utilizá-la em situações reais, que é um trabalho futuro. Estas variáveis são analisadas e discutidas através de experimentos que comprovam a eficiência e eficácia da abordagem proposta e sua importância no apoio à construção de sistemas de ensino.

Para cada experimento define-se os estilos de aprendizagem reais e a força de cada preferência (forte, moderada, fraca ou balanceada). Para cada experimento, apresenta-se graficamente o processo de atualização das probabilidades durante o processo de aprendizagem. Em cada gráfico, o eixo $x$ mostra o número de iterações do processo de aprendizagem, e o eixo $y$ mostra os valores que os estilos de aprendizagem probabilísticos assumem ao longo do processo de aprendizagem em cada dimensão.

O objetivo principal foi observar como o modelo do estudante é gradualmente atualizado ao longo de cada seção (ou iteração) do processo de aprendizagem. Desta forma, foi possível visualizar claramente o processo de detecção e correção automática de estilos de aprendizagem em cada uma das dimensões do FSLSM.

Também, apresenta-se graficamente a variação da média da performance e da quantidade de problemas de aprendizagem ocorridos, em intervalos de 20 iterações. Isto permite verificar como a performance média aumenta e a quantidade de problemas de aprendizagem diminui à medida que o modelo do estudante é corrigido e se torna consistente em relação aos estilos de aprendizagem reais do estudante. Este experimento considerou um estudante com EAp inicializados conforme apresenta a Tabela 4.

\begin{tabular}{|c|c|c|c|c|c|c|c|}
\hline \multicolumn{7}{|c|}{ Estilos de Aprendizagem Probabilisticos (EAp) } \\
\hline \multicolumn{2}{|c|}{ Processamento } & \multicolumn{2}{|c|}{ Percepção } & \multicolumn{2}{c|}{ Entrada } & \multicolumn{2}{c|}{ Organização } \\
\hline Ativo & Reflex. & Sens. & Intuit. & Visual & Verbal & Seq. & Global \\
\hline 0,7 & 0,3 & 0,7 & 0,3 & 0,7 & 0,3 & 0,7 & 0,3 \\
\hline
\end{tabular}

Tabela 4. Estilos de Aprendizagem Probabilísticos

Os estilos de aprendizagem reais do estudante considerado são: Reflexivo(forte), Intuitivo (forte), Verbal (moderado), Global (leve). Considerando um estu- 
dante com este perfil, os valores apresentados na Tabela 4 são inconsistentes em suas 4 dimensões.

A Figura 4 apresenta graficamente o processo de atualização das probabilidades durante a primeira execução deste experimento. É importante notar que os estilos de aprendizagem probabilísticos se tornaram consistentes com os reais em todas as suas dimensões, satisfazendo os aspectos de consistência e eficiência esperados.

A Tabela 5 apresenta o número de iterações, a quantidade de problemas de aprendizagem ocorridos, e os valores dos EAp's resultantes ao final do processo de aprendizagem simulado em 10 execuções consecutivas deste experimento. Pode-se verificar que em todas as repetições do processo os EAp ficaram consistentes em relação aos EA reais do estudante.

\begin{tabular}{llll}
\hline $\mathbf{N}$. & Iterações & Prob. Apr. & Resultados \\
\hline 1 & 208 & 28 & $\{(0.2 ; 0.8) ;(0.1 ; 0.9) ;(0.1 ; 0.9) ;(0.3 ; 0.7)\}$ \\
\hline 2 & 204 & 24 & $\{(0.2 ; 0.8) ;(0.1 ; 0.9) ;(0.2 ; 0.8) ;(0.3 ; 0.7)\}$ \\
\hline 3 & 213 & 33 & $\{(0.2 ; 0.8) ;(0.2 ; 0.8) ;(0.2 ; 0.8) ;(0.1 ; 0.9)\}$ \\
\hline 4 & 206 & 26 & $\{(0.1 ; 0.9) ;(0.2 ; 0.8) ;(0.2 ; 0.8) ;(0.2 ; 0.8)\}$ \\
\hline 5 & 201 & 21 & $\{(0.2 ; 0.8) ;(0.2 ; 0.8) ;(0.2 ; 0.8) ;(0.2 ; 0.8)\}$ \\
\hline 6 & 204 & 24 & $\{(0.2 ; 0.8) ;(0.2 ; 0.8) ;(0.2 ; 0.8) ;(0.1 ; 0.9)\}$ \\
\hline 7 & 210 & 30 & $\{(0.1 ; 0.9) ;(0.2 ; 0.8) ;(0.2 ; 0.8) ;(0.3 ; 0.7)\}$ \\
\hline 8 & 196 & 16 & $\{(0.1 ; 0.9) ;(0.2 ; 0.8) ;(0.2 ; 0.8) ;(0.2 ; 0.8)\}$ \\
\hline 9 & 200 & 20 & $\{(0.2 ; 0.8) ;(0.1 ; 0.9) ;(0.1 ; 0.9) ;(0.2 ; 0.8)\}$ \\
\hline 10 & 198 & 18 & $\{(0.2 ; 0.8) ;(0.3 ; 0.7) ;(0.1 ; 0.9) ;(0.3 ; 0.7)\}$ \\
\hline Med. & 204 & 24 & - \\
\hline
\end{tabular}

Tabela 5. Resultados obtidos em 10 execuções consecutivas do experimento
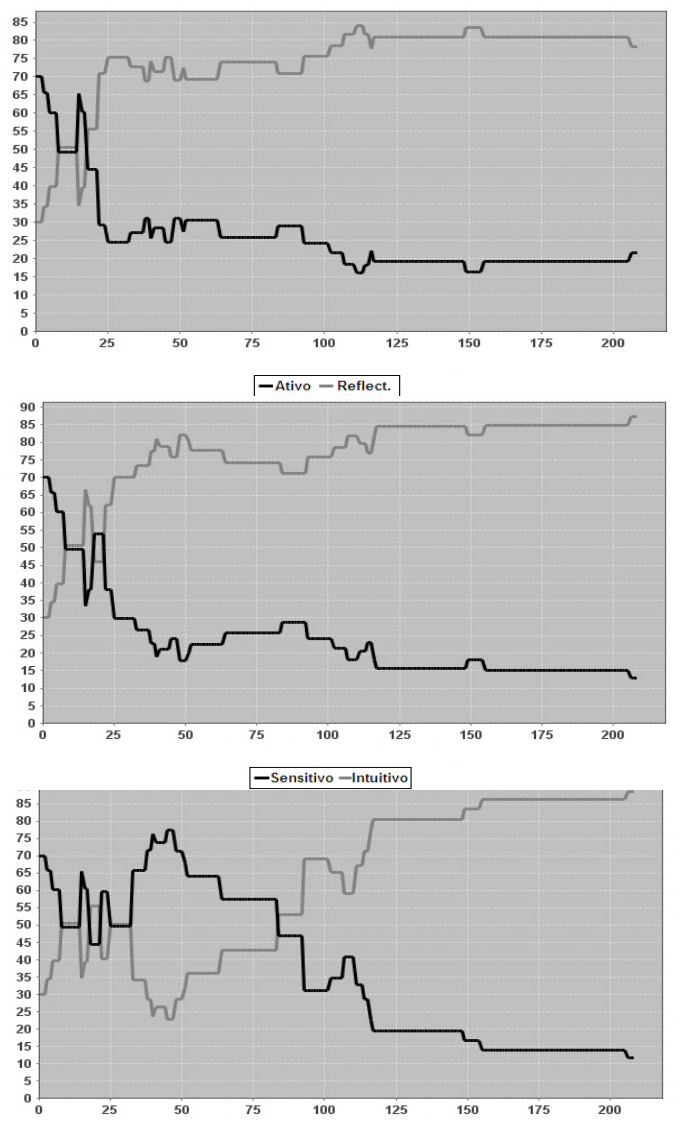

Figura 4. Atualização dos EAp's do estudante durante o processo de aprendizagem.

A Figura 5 apresenta a performance média alcançada pelo estudante e a média de problemas de aprendizagem a cada 20 iterações do processo. Pode-se perceber que mesmo quando os estilos de aprendizagem probabilísticos são consistentes com os reais (ou seja, $d=0$ na equação (2)), problemas de aprendizagem podem ocorrer, já que outros fatores exercem influência na performance do estudante.

Mas, fica claro que a performance média do estudante se eleva gradualmente à medida que seus EAp se tornam consistentes com suas preferências reais. Além disto, podemos observar que estes resultados atendem aos requisitos de consistência e eficiência inicialmente propostos nesta seção, já que os estilos de aprendizagem probabilísticos efetivamente convergiram para os estilos de aprendizagem reais do estudante, e tornam-se consistentes no início do processo de aprendizagem, demonstrando a eficiência do processo, mesmo quando há fortes inconsistências no EAp do estudante.

Apresenta-se a seguir os resultados relativos as 9 primeiras iterações em um experimento realizado com o modelo proposto neste trabalho para personalização do conteúdo, implementado através dos algoritmos apresentados na seção anterior.

No ME inicial do estudante, os EAp foram inicializados em $50 \%$ para todos os EA, em todas as dimensões, ou seja, nenhuma preferência foi inicialmente detectada. A medida que o processo evolui, o sistema atualiza as preferências do estudante, conforme apresentado na seção anterior. Este experimento considerou um estudante com EAp inicializados conforme apresenta a Tabela 6. 

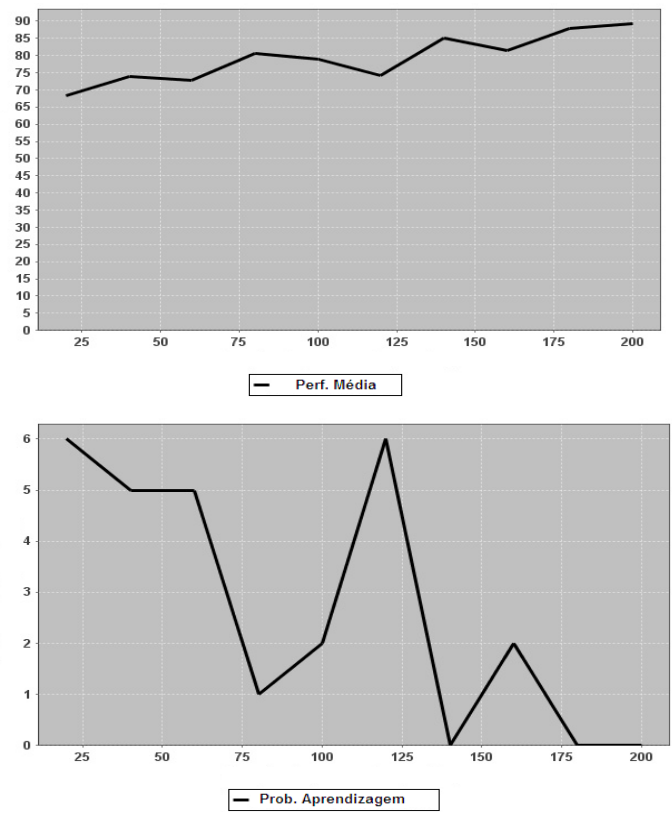

Figura 5. Performance média e problemas de aprendizagem ao longo do processo

\begin{tabular}{|c|c|c|c|c|c|c|c|}
\hline \multicolumn{7}{|c|}{ Estilos de Aprendizagem Probabilisticos (EAp) } \\
\hline \multicolumn{2}{|c|}{ Processamento } & \multicolumn{2}{|c|}{ Percepção } & \multicolumn{2}{c|}{ Entrada } & \multicolumn{2}{c|}{ Organização } \\
\hline Ativo & Reflex. & Sens. & Intuit. & Visual & Verbal & Seq. & Global \\
\hline 0,5 & 0,5 & 0,5 & 0,5 & 0,5 & 0,5 & 0,5 & 0,5 \\
\hline
\end{tabular}

Tabela 6. Estilos de Aprendizagem Probabilísticos

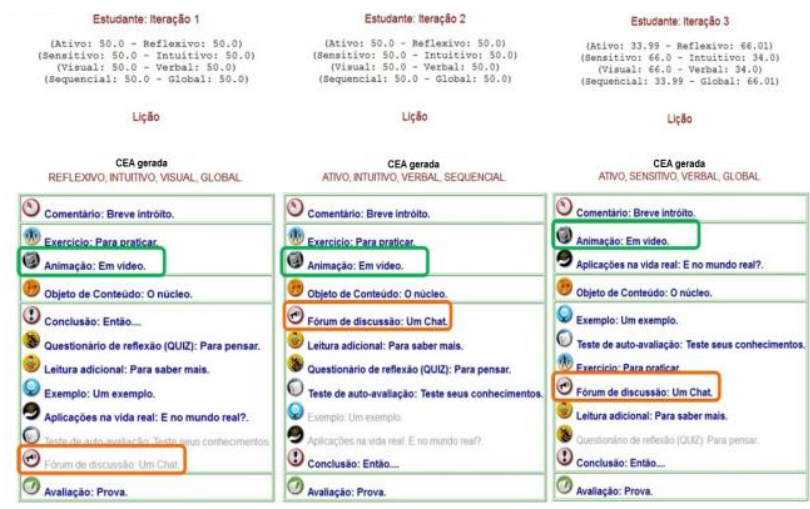

Figura 6. Adaptatividade gerada nas iterações 1 a 3

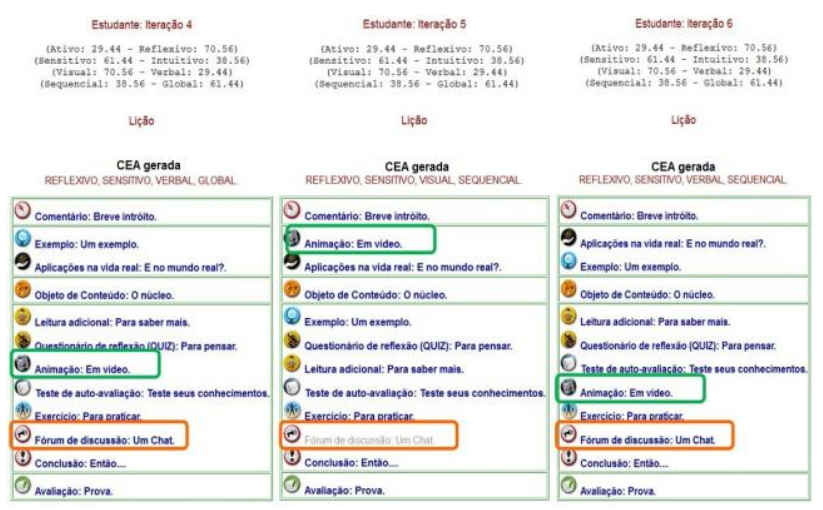

Figura 7. Adaptatividade gerada nas iterações 4 a 6

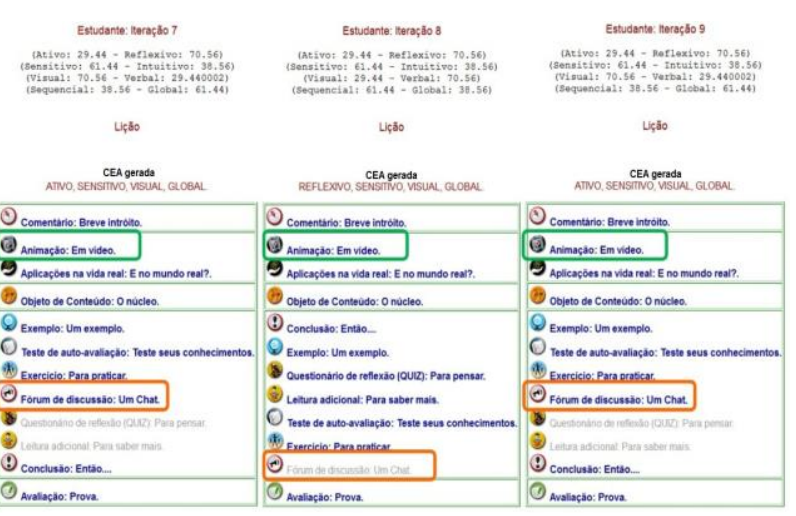

Figura 8. Adaptatividade gerada nas iterações 7 a 9

A cada iteração, é apresentada a recomendação de conteúdo produzida pela abordagem proposta, assim como o valor dos EAp do estudante. A Figura 6 apresenta os resultados obtidos nas iterações 1 a 3 . A Figura 7 apresenta os resultados obtidos nas iterações 4 a 6 . A Figura 8 apresenta os resultados obtidos nas iterações 7 a 9 .

Uma das características fundamentais desse processo é o comportamento estocástico. Assim, percebemos que nem sempre o EA de maior probabilidade no ME é selecionado para compor a CEA, e então, não é considerado na adaptatividade gerada.

Isto pode ser facilmente percebido, por exemplo, na iteração 6, Figura 7, quando foram considerados, na geração de adaptatividade, os estilos verbal e sequencial, os quais não eram, naquela iteração, os estilos de maior probabilidade em suas respectivas dimensões no ME. Vale lembrar que isso ocorre porque, na abordagem proposta, não se considera o EAp maior como sendo uma preferência definitiva, de forma determinística, mas sim como uma tendência. Isso permite que, em caso de inconsistências, as preferências corretas possam ser detectadas, tornando o processo de adaptatividade mais efetivo.

Destaca-se, ainda, ao longo das iterações apresentadas, a evolução do posicionamento de alguns objetos. O objeto Animação é recomendado como primeiro objeto da área 1. Ao longo das iterações, a posição designada para esse objeto variou, chegando a aparecer entre os últimos conteúdos recomendados (iterações 4 e 6). Entretanto, à medida que os valores dos EAp do estudante convergem para as suas preferências reais, o objeto Animação passa a ocupar sua posição adequada.

Da mesma forma, o objeto Fórum de discussão, chegou a ser o primeiro objeto da área 2 (iteração 2). Ao 
longo do processo, este também passou a ocupar uma posição mais adequada, considerando-se os EAp do estudante.

Conclui-se, então, que a abordagem apresentada neste trabalho mostrou-se completamente aderente À geração de adaptatividade para estudantes modelados através de uma abordagem automática, dinâmica e estocástica de EA. A abordagem apresentada, mesmo com um pequeno número de iterações aplicado, já demonstrou eficácia na modelagem do estudante, a adaptatividade gerada condiz com o que seria esperado para ambos os casos.

Finalmente, acredita-se que os resultados obtidos por experimentos validam a abordagem proposta neste trabalho, estando então apta a ser implementada e utilizada em um ambiente existente, em um processo de ensinoaprendizagem real. A próxima seção tece conclusões e aponta trabalhos futuros.

\section{Conclusão e trabalhos futuros}

A experimentação da abordagem proposta apresentou resultados promissores, como demonstrado na seção anterior. Os testes realizados apresentaram uma organização dos OAs da forma eficiente, levando em consideração os relacionamentos estabelecidos por [3] entre os OAs.

A abordagem apresentada utiliza um processo não-determinístico de modelagem do estudante e de recomendação de OAs, baseado na seleção estocástica de combinações de estilos de aprendizagem de acordo com as probabilidades de preferências armazenadas no modelo do estudante. Como os estilos de aprendizagem sofrem alterações ao longo do tempo, nota-se claramente que este processo é de natureza não-determinística.

Um processo baseado em aprendizagem por reforço é responsável por atualizar constantemente os EAp do estudante, que evoluem de forma iterativa e incremental ao longo do processo de aprendizagem. Desta forma, as preferências por EA do estudante podem ser automaticamente detectadas, quando não se tem informações sobre elas, ou mesmo corrigidas, quando estiverem inconsistentes. Este processo atualiza os EAp do estudante sempre que um desempenho insatisfatório é detectado.

A validação do modelo proposto através de técnicas de simulação computacional utilizando-se perfis fictícios de estudantes foi fundamental, já que a implantação de projetos de e-learning requerem quantias consideráveis de recursos financeiros, humanos e de tempo [19].
Desta forma, é de fundamental importância que se possa avaliar a performance de um modelo antes de implantá-lo efetivamente em um LMS (Learning Management System), devido à complexidade desta tarefa e da quantidade de recursos exigidos. Esta questão é ainda mais crítica quando se considera que a demanda por sistemas de e-learning é cada vez maior, e o número de estudantes que dependem destes sistemas cresce rapidamente [19].

Então, foi possível observar a eficiência da abordagem proposta, principalmente quando da necessidade de se oferecer um curso personalizado, em ambiente de hipermídia adaptativa, a um grande número de estudantes. Desta forma, como trabalho futuro, tem-se a implantação deste método em um LMS existente, tal como o Moodle [2], para que possa ser testado com estudantes reais.

\section{Referências}

[1] W. C. B. Neto (2005) "WEB Semântica, Sistemas Adaptativos e Educação a Distância: Propostas e Perspectivas". Universidade do Planalto Catarinense. Conferência IADIS IberoAmericana WWW/Internet 2005.

[2] Moodle. (2013). Disponível em $<$ https://moodle.org/>. Acesso em 06 de janeiro de 2013.

[3] S. Graf; Kinshuk; C. Ives (2010). A Flexible Mechanism for Providing Adaptivity Based on Learning Styles in Learning Management Systems. Athabasca University, Canada.

[4] TelEduc. (2013). Disponível em: $<$ http://www.teleduc.org.br/>. Acesso em 06 de janeiro de 2013.

[5] P. Brusilovsky (1996b). A Tool for Developing Adaptive Electronic Textbooks on WWW. School of Computer Science, Carnegie Mellon University, Pittsburgh.

[6] P. Brusilovsky (1996a). Methods and techniques of adaptive hypermedia. Seventh International PEG Conference, Edinburgh.

[7] I. Bittencourt; E. Costa (2011). Modelos e Ferramentas para a Construção de Sistemas Educacionais adaptativos e Semânticos. Universidade Federal de Alagoas. 
[8] M. Nat; S. Walker; L. Babcon; M. Dastbaz (2010). Designing personalisation in LAMS. University of Greenwich.

[9] R. Felder; L. Silverman (1988). Learning and teaching styles in engineering education. Engineering Education, vol. 78, pp. 674-681.

[10] F. A. Dorça; L. V. Lima; M. A. Fernandes; C. R. Lopes (2012). A Stochastic Approach for Automatic and Dynamic Student Modeling in Adaptive Educational Systems. Informatics in Education. Volume 11, Número 2.

[11] S. Graf (2007). Adaptivity in Learning Management Systems Focussing on Learning Styles. Vienna University of Technology.

[12] R. Felder; B. Soloman (2006). Index of Learning Style Questionnaire. North Carolina State University. Disponível em $<$ http://www4.ncsu.edu/unity/lockers/users/f/feld er/public/ILSpage.html>. Acesso em 20 set. 2012, 22:40.

[13] P. Paredes; P. Rodrigues (2001). Considering Sensing-Intuitive Dimension to ExpositionExemplification in Adaptive Sequencing. Escuela Técnica Superior de Informática, Madrid, Espanha.

[14] F. A. Dorça; L. V. Lima; M.A. Fernandes; C. R. Lopes, (2011) A New Approach to Discover Students Learning Styles in Adaptive Educational Systems. Anais do XXII SBIE - XVII WIE Aracaju, 21 a 25 de novembro de 2011.

[15] F. A. Dorça; L. V. Lima; M. A. Fernandes; C. R. Lopes (2013) Comparing strategies for modeling students learning styles through reinforcement learning in adaptive and intelligent educa- tional systems: An experimental analysis. Expert Systems with Applications 40 (2013) 20922101.

ttp://dx.doi.org/10.1016/j.eswa.2012.10.014

[16] H. Wu (2002). A reference architeture for adaptive hypermedia applications. University Press Facilities, Eindhoven, the Netherlands.

[17] P. Brusilovsky (2001). Adaptive Educational Hypermedia. School of Information Science, University of Pittsburgh.

[18] L. S. Munoz; J. P. Oliveira (2004). Adaptive Web-Based Courseware Development using Metadata Standards and Ontologies. Universidade Federal do Rio Grande do Sul, Porto Alegre.

[19] S. Graf ; C. Lan, T. Liu, et al. (2009). "Investigations about the Effects and Effectiveness of Adaptivity for Students with Different Learning Styles," in 2009 Ninth IEEE International Conference on Advanced Learning Technologies, pp. 415-419, IEEE. 